\title{
STABILITY OF BOVINE CYTOCHROME $C$ OXIDASE AS STUDIED AFTER EXPOSURE TO HIGH HYDROSTATIC PRESSURE
}

\author{
Jana Staničová ${ }^{1}$, Andrej Musatov ${ }^{2}$, Neal C. Robinson ${ }^{2}$ \\ University of Veterinary Medicine, Košice, Slovak Republic: Institute of Chemistry, Biochemistry, and Biophysics ${ }^{1}$; The \\ University of Texas Health Science Center, San Antonio, Texas 78229-3900, USA: Department of Biochemistry ${ }^{2}$

\begin{abstract}
Summary: Structural and functional stability of bovine cytochrome $c$ oxidase as a function of exposure to high hydrostatic pressure is reported. The pressure affects the stability of monomeric and dimeric enzyme quite differently. Exposure of the monomeric cytochrome $c$ oxidase to pressures higher than 2.5 kbar causes dissociation of subunits III, VIa, VIb, VIIa with a $35-50 \%$ decrease in electron transport activity. Dimeric enzyme is more resistant to high hydrostatic pressure since subunits III and VIIa do not dissociate and the electron transport activity loss is minimal.
\end{abstract}

Key words: Cytochrome c oxidase; High hydrostatic pressure; Dimer; Monomer; Electron transport activity; Subunit dissociation

\section{Introduction}

Cytochrome $c$ oxidase $\left(\mathrm{CcO}^{\S}\right.$; Ec 1.9.3.1) is the terminal enzyme of the inner mitochondrial electron transport chain (18). Bovine heart $\mathrm{CcO}$ is consisted of 13 different subunits (6) and the enzyme is almost always dimeric in three-dimensional lattice as it was obtained from crystallographic data (15).

The three largest subunits (I-III) are encoded by mitochondrial DNA and are synthesized in the mitochondria. They constitute the functional core of the enzyme and include all of the redox-active centers. The smaller subunits (IV-VIII) are nuclear encoded and most likely have a structural and/or regulatory role $(4,5)$.

Treatment of $\mathrm{CcO}$ with physical (high hydrostatic pressure) or chemical (chaotropic agents, e.g., urea - 13, peroxidation by hydrogen peroxide - 9) factors enables to study the structural effects. Consequently, the relation between structural changes and enzymatic activity may be determined. We report the effects of hydrostatic pressure on electron transport activity and structural composition of $\mathrm{CcO}$. Our approach is the study of pressure influence on $\mathrm{CcO}$ in view of different behavior of dimeric and monomeric $\mathrm{CcO}$ forms.

\section{Materials and methods}

Materials. Bovine cytochrome $c$ oxidase was prepared from Keilin-Hartree heart particles by the method of Fowler et al. (2) with modifications described by Mahapatro and Robinson (8). The final oxidase pellet was solubilized in $100 \mathrm{mM}$ phosphate buffer, $\mathrm{pH} 7.4$ containing $1 \%$ sodium cholate and $1 \mathrm{mM}$ EDTA. Individual drops of purified enzyme $(25 \mathrm{mg} / \mathrm{mL}$ protein) were quickly frozen by pipetting the solution into liquid nitrogen and stored at $-80{ }^{\circ} \mathrm{C}$. Cytochrome $c$ oxidase concentrations were calculated on the basis of $\epsilon_{422}=1.54 \times 10^{5} \mathrm{M}^{-1} \mathrm{~cm}^{-1}$ (16). Horse heart cytochrome $c$ (Type III) was purchased from Sigma Chemical Co. Reduced cytochrome $c$ was freshly prepared by dithionite reduction, and an excess dithionite was removed by G-25 Sephadex gel filtration. Initial concentrations of ferrocytochrome $c$ were determined using $\epsilon_{550}=21 \mathrm{mM}^{-1} \mathrm{~cm}^{-1}$. All other chemicals were reagent grade.

Methods. The high hydrostatic pressure experiments were performed as described in (11). Kinetics measurements and fluorescence spectra were recorded, respectively. In case of kinetics measurements tryptophan fluorescence was monitored $\left(\lambda_{\mathrm{ex}}=295 \mathrm{~nm}, \lambda_{\mathrm{em}}=326 \mathrm{~nm}\right)$ as a function of time. Fluorescence spectra of tryptophan were recorded in emission range $300-500 \mathrm{~nm}$ using excitation wavelength $295 \mathrm{~nm}$.

Determination of cytochrome c oxidase activity. Molecular activity of $\mathrm{CcO}$ was assayed spectrophotometrically at $25^{\circ} \mathrm{C}$ with $25-30 \mu \mathrm{M}$ ferrocytochrome $c$ as a substrate in $25 \mathrm{mM}$ phosphate buffer, $\mathrm{pH} 7.0$, containing $1 \mathrm{mg} / \mathrm{mL}$ dodecyl maltoside. Molecular activities were calculated by the pseudo-first-order rate of oxidation of ferrocytochrome $c$ by

\$Abbreviations: $\mathrm{CcO}$, bovine heart cytochrome $c$ oxidase; EDTA, ethylenediaminetetraacetic acid; FPLC, fast performance liquid chromatography; HPLC, high performance liquid chromatography; SDS-PAGE, sodium dodecyl sulfate polyacrylamide gel electrophoresis 
$1.75 \mathrm{nM}$ cytochrome $c$ oxidase (1). The activity of the purified enzyme was $300-350 \mathrm{~s}^{-1}$ to an accuracy of $\pm 5 \%$.

HiTrapQ FPLC ion exchange chromatography. Anion exchange chromatography on the HiTrapQ column was a modification of the method developed for a MonoQ FPLC column (7) and was used as described in (7).

Analysis of subunits. Nuclearly encoded subunits (subunits IV-VIII, nomenclature according to Kadenbach et al. (6) were determined by $\mathrm{C}_{18}$ reversed phase HPLC (7). Absorbance intensity of subunit $\mathrm{Vb}$ was taken as the intensity standard for the chromatogram normalization. Determination of three mitochondrially encoded subunits (subunits I-III) was performed by SDS-polyacrylamide gel electrophoresis on $15 \%$ acrylamide gels that contained $2 \mathrm{M}$ urea in addition to $0.1 \%$ SDS (12). Subunit content was determined by scanning on Personal Densitometer SI (Molecular Dynamics) using ImageQuant version 5.2 software.

\section{Results}

Monomeric and dimeric $\mathrm{CcO}$ were prepared by manipulating the concentrations of dodecyl maltoside and sodium cholate; high concentrations of dodecyl maltoside (5-10 $\mathrm{mM})$ produce monomeric $\mathrm{CcO}$ while a mixture of dodecyl maltoside and sodium cholate produce dimeric $\mathrm{CcO}[10]$. Homogeneity of the monomeric or dimeric forms was confirmed by sedimentation velocity as previously described (17).
Effect of high pressure on the electron transport activity of cytochrome $c$ oxidase. Molecular activity of dimeric and monomeric $\mathrm{CcO}$ was assayed spectrophotometrically and obtained results showed a significant difference in the pressure-induced effect of dimer, and monomer, respectively. Exposure of dimeric $\mathrm{CcO}$ to pressure causes minimal loss of activity of enzyme (7-13\% at the pressures $2.00-2.75$ kbar)) (Fig.1). On the other hand the same pressures induce remarkable decrease in activity of monomeric $\mathrm{CcO}$ (24-48\%) (Fig.1). From these results we can conclude that dimeric $\mathrm{CcO}$ is functionally more stable than monomeric one if both forms are exposed to the equal hydrostatic pressure.

Effect of high pressure on the structural composition of cytochrome c oxidase. We used HiTrapQ ion exchange chromatography, combined with $\mathrm{C}_{18}$ reversed-phase HPLC and SDS-PAGE for quantitatively assessing a subunit content of $\mathrm{CcO}$. Chromatographic elution of purified $\mathrm{CcO}$ from a HiTrapQ FPLC ion exchange column allows a separation of intact 13-subunit $\mathrm{CcO}$ complex from a form that is missing subunits (7). Figure 2a shows HiTrapQ FPLC elution of dimeric $\mathrm{CcO}$ after exposure to high hydrostatic pressure 2.5 kbar. Protein eluted as the peak A and a small peak B without a significant difference between pressure-treated $\mathrm{CcO}$ and control sample (Fig. 2a). The gel electrophoresis and $\mathrm{C}_{18}$ HPLC chromatography confirmed that peak A contains intact 13-subunit complex and peak B contains 11 subunits and is completely devoid of subunit VI and VIb.

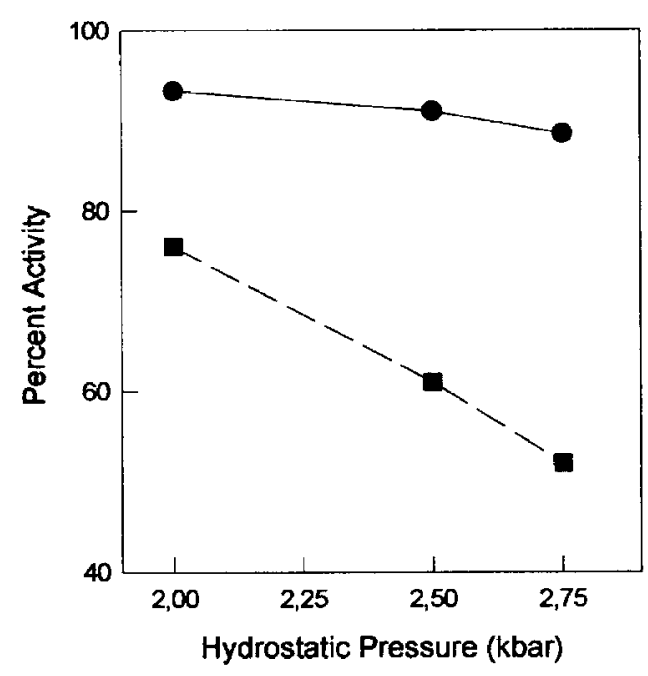

Fig. 1: Dependence of dimeric and monomeric cytochrome $c$ oxidase activity on high hydrostatic pressure. Dimeric $\mathrm{CcO}(5 \mu \mathrm{M})$ (circles) was prepared in $20 \mathrm{mM}$ Tris-Cl pH 7.4 with $1 \mathrm{mg} / \mathrm{mg}$ protein dodecyl maltoside in absence of dialysis. Monomeric $\mathrm{CcO}(5 \mu \mathrm{M})$ (squares) was prepared with $5 \mathrm{mg} / \mathrm{mg}$ protein dodecyl maltoside followed by dialysis overnight in $20 \mathrm{mM}$ Tris- $\mathrm{Cl} \mathrm{pH} 7.4$ with $0.1 \mathrm{mg} / \mathrm{mL}$ dodecyl maltoside.

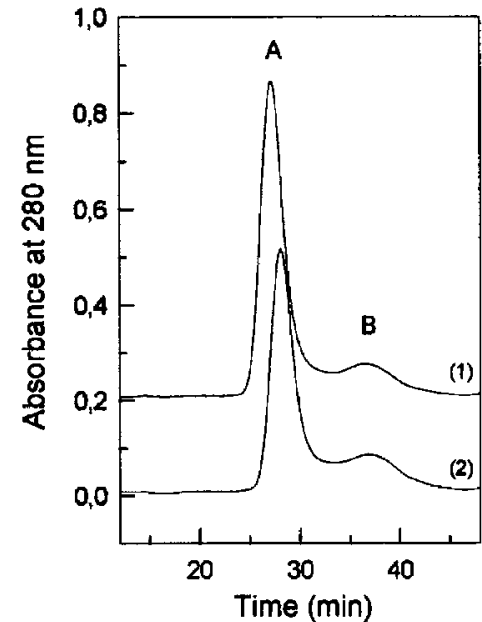

(a)

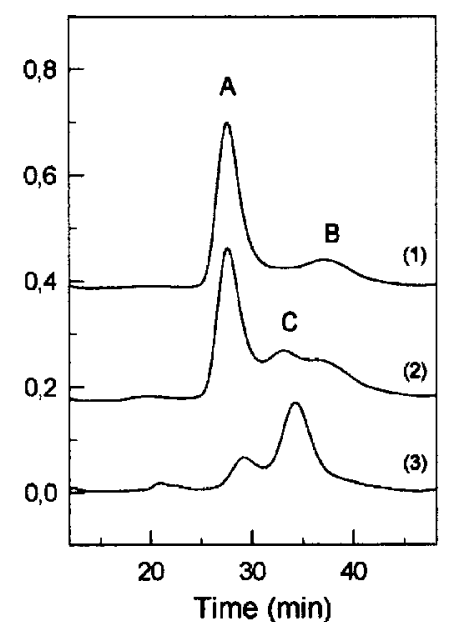

(b)
Fig. 2: HiTrapQ FPLC ion exchange chromatography elution of dimeric (a) and monomeric (b) cytochrome $c$ oxidase as measured after exposure to high hydrostatic pressure. Both forms were prepared as described in Fig. 1. The elution gradient was as described previously (7). HiTrapQ elution of dimer (a) after exposure to $2.5 \mathrm{kbar}$ is represented by trace 2 . Trace 1 shows the elution of control sample. The elution of monomer (b) after exposure to $2.00 \mathrm{kbar}$ is represented by trace 2 , and to $2.5 \mathrm{kbar}$ by trace 3 . Trace 1 shows elution of sample in absence pressure. 
Different situation became when the monomeric $\mathrm{CcO}$ was exposed to high hydrostatic pressure (Fig. 2b). Increase of pressure to $2.5 \mathrm{kbar}$ caused a remarkable change in the chromatographic elution of monomeric $\mathrm{CcO}$ (Fig. $2 \mathrm{~b}$ trace 3 ). As it can be seen from figure, the peak $\mathrm{C}$ was the most intensive and the peak B completely disappeared.

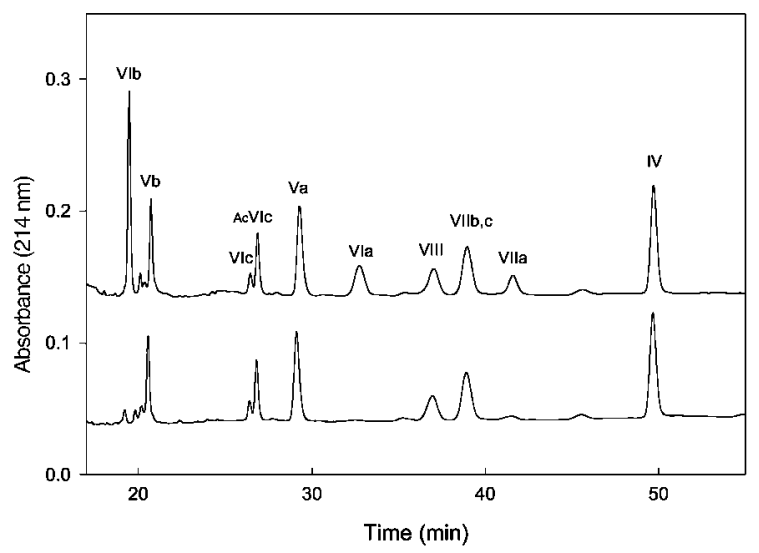

Fig. 3: Subunit content of two forms of $\mathrm{CcO}$ after exposure to high hydrostatic pressure as determined by reversed phase HPLC. Upper trace: subunit content of HiTrapQ Peak A, which is the intact 13 -subunit $\mathrm{CcO}$. Lower trace: subunit content of HiTrapQ Peak C, which is the subunit depleted form of $\mathrm{CcO}$. The method quantitatively determines the content of the 10 nuclearly encoded subunits (subunits IV through VIII), but does not detect the mitochondrially encoded subunits I, II and III (refer to Fig. 4 for their analysis).
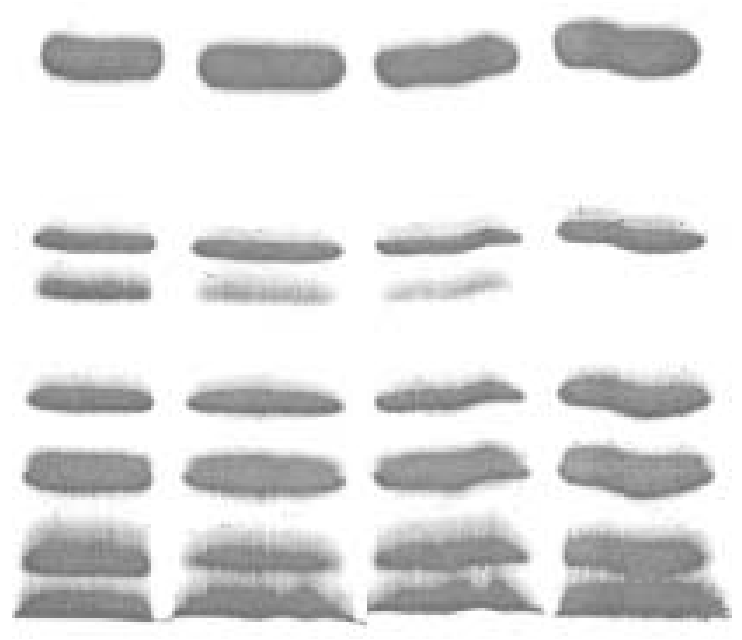

Fig. 4: SDS-PAGE subunit content of HiTrapQ elution peak A (13-subunit intact form) of pressure-untreated monomeric $\mathrm{CcO}$ (column 1), peak A of pressure-treated (2.5 kbar) monomeric $\mathrm{CcO}$ (column 2), peak B (11-subunit form) of pressure-untreated $\mathrm{CcO}$ (column 3), and peak $\mathrm{C}$ of pressure-treated $\mathrm{CcO}$ (column 4). Conditions SDSPAGE are described in Methods.
Reversed-phase $\mathrm{C}_{18}$ HPLC enables to determine the 10 nuclear encoded subunits (IV - VIII), which are very difficult to separate by SDS - PAGE (7). We used this method for a determination of monomeric $\mathrm{CcO}$ subunit composition of eluting protein shown in Fig. 2b (peaks A, C) (Fig. 3).

The chromatographic analysis of the peak $\mathrm{C}$ shows a loss of subunits VIa, VIb, VIIa (Fig. 3). Content of subunit VIIa was only $17 \%$ from that in the absence pressure and the intensity of subunits VIa (2\%) and VIb (8\%) decreased enormously (Fig. 3). Similar results were obtained when monomeric $\mathrm{CcO}$ was exposed to $2.75 \mathrm{kbar}$ pressure. From our findings, we can conclude that the most of the monomeric $\mathrm{CcO}$ exposed to pressure $2.5 \mathrm{kbar}$ and higher values occurs in the form, which is missing subunits VIa, VIb and VIIa (Figures $2 b$ and 3 ).

Determination and quantitation of the three largest subunits (I - III) was provided by SDS-PAGE. The composition of the monomeric $\mathrm{CcO}$ exposed to $2.5 \mathrm{kbar}$ eluting in the HiTrap elution as peaks A, C (Fig. 2b, trace 3) as well as the control enzyme resulting in peaks $\mathrm{A}, \mathrm{B}$ is shown in Fig. 4, columns 1-4.

The intact 13 -subunit control $\mathrm{CcO}$ eluted as peak $\mathrm{A}$ is presented in column 1 in Fig. 4 and the intact 13-subunit complex of $\mathrm{CcO}$ exposed to $2.5 \mathrm{kbar}$ is shown in column 2 . Although we can see a small difference in intensity of subunit III band between control sample and sample exposed to pressure, the subunit III is $96 \%$ the control sample. Remarkable change was recorded in case of peak C. Subunit III band of the pressure treated enzyme completely disappeared (Fig. 4, column 4) and the percent of this subunit is only $6 \%$ the control CcO. From results obtained using SDS-PAGE it is clear that not only subunits VIa, VIb, VIIa but also subunit III is missing in peak $\mathrm{C}$ elution of monomeric $\mathrm{CcO}$. We can conclude: the exposure of monomeric $\mathrm{CcO}$ to pressure higher than $2.5 \mathrm{kbar}$ leads to changes of its composition and therefore, disturbs a structural integrity of monomeric enzyme. With dimeric enzyme, only subunits VIa and VIb dissociate, neither of which is essential for full electron transport activity.

\section{Discussion}

High hydrostatic pressure was used as a physical factor for a study of possible different behavior of dimeric and monomeric $\mathrm{CcO}$. Both forms of $\mathrm{CcO}$ were estimated from point of view of its partial functional stability (pressure effect on redox- state of $\mathrm{CcO}$ (unpublished results) and electron transport activity; proton pumping has not been estimated) and structural stability (pressure effect on subunit dissociation). We found that high hydrostatic pressure affects the structural and functional stability of monomeric and dimeric $\mathrm{CcO}$ quite differently.

The chemical effects on the structure and enzymatic activity of dimeric $\mathrm{CcO}$ were investigated $(9,13,14)$. Treat- 
ment of dimeric $\mathrm{CcO}$ with chemical factors (removal of cardiolipin -14 , treatment with urea -13 , hydrogen peroxide -9) has important consequences on the structure and function of dimeric enzyme. From our results and considering findings in (14) we can suppose partial dissociation of subunits VIa and VIb due to hydrostatic pressure, which is finished on chromatographic column. In accordance with (14), the decrease in electron transport activity of dimeric $\mathrm{CcO}$ due to high pressure was not significant. The exposure of dimeric $\mathrm{CcO}$ to chemical factors has more consequences than the exposure to hydrostatic pressure. Possible explanation can be different acting of the pressure. In summary, dimeric form of enzyme is very stable when exposed to high hydrostatic pressure.

The different situation is when the monomeric $\mathrm{CcO}$ is treated with high hydrostatic pressure. The exposure of monomeric enzyme to hydrostatic pressure causes dissociation of subunits III, VIa, VIb, VIIa with a significant decrease in electron transport activity. Also the percent of reduction is higher when monomer is exposed to pressure. The subunits VIa and VIb are not responsible for the loss of activity (14) of enzyme. It is known that the dissociation of core subunit III does not alter electron transport activity of $\mathrm{CcO}$ (3). From our results we can suppose that the dissociation of subunit VIIa causes significant decrease in enzymatic activity of monomeric $\mathrm{CcO}$. These findings are consistent with results obtained by exposure of $\mathrm{CcO}$ to urea (13).

In summary, we can conclude that high hydrostatic pressure perturbs functional (electron transport activity) and structural integrity of monomeric $\mathrm{CcO}$, while dimeric form is more resistant to pressure. Monomeric form of $\mathrm{CcO}$ is more fragile than dimeric $\mathrm{CcO}-$ this fact can contribute to the general assumption that dimeric form of $\mathrm{CcO}$ occurs in vivo.

\section{Acknowledgements}

This work was supported by a research grants from National Institutes of Health (NIH GMS 24795) and The Robert A. Welch Foundation (AQ 1481)

The authors especially wish to thank Dr. Mark Panda for his help with the pressure experiments and Ms. Linda Sowdal for technical assistance.

\section{References}

1. Dale MP, Robinson NC. Synthesis of cardiolipin derivatives with protection of the free hydroxyl: its application to the study of cardiolipin stimulation of cytochrome c oxidase. Biochemistry 1988;27:8270-5.

2. Fowler LR, Richardson SH, Hatefi Y. A rapid method for the preparation of highly purified cytochrome oxidase. Biochim Biophys Acta 1962;64:170-3.

3. Haltia T, Saraste M, Wikstrőm, M. Subunit-III of cytochrome-c-oxidase is not involved in proton translocation - a site -directed mutagenesis study. EMBO J 1991; 10:2015-21.

4. Kadenbach B. Regulation of respiration and ATP synthesis in higher organisms. J Bioenerg. Biomembr. 1986;18:39-54

5. Kadenbach B, Merle P. On the function of multiple subunits of cytochrome c oxidase from higher eukaryotes. FEBS Lett 1981;135:1-11.

6. Kadenbach B, Jaraush J, Hartmann R, Merle P. Separation of mammalian cytochrome c oxidase into 13 polypeptides by a sodium sulfate-gel electrophoretic procedure. Anal Biochem 1983;129:517-21

7. Liu YC, Sowdal L, Robinson NC. Separation and quantitation of cytochrome c oxidase subunits by Mono-Q fast protein liquid chromatography and C18 reversephase high-performance liquid chromatography. Arch Biochem Biophys 1995; $324: 135-42$

8. Mahapatro SN, Robinson NC. Effect of changing the detergent bound to bovine cytochrome c oxidase upon its individual electron-transfer steps. Biochemistry 1990;29:764-70.

9. Musatov A, Hebert E, Carrol CA, Weintraub ST, Robinson NC. Specific modification of two tryptophans within the nuclear-encoded subunits of bovine cytochrome c oxidase by hydrogen peroxide. Biochemistry 2004;43:1003-9.

10. Musatov A, Ortega-Lopez J, Robinson NC. Detergent-solubilized bovine cytochrome c oxidase: dimerization depends on the amphiphilic environment. Biochemistry 2000:39:12996-13004.

11. Panda M, Ybarra J, Horowitz PM. Dissociation of the single-ring chaperonin GroEL by high hydrostatic pressure. Biochemistry 2002;41:12843-9.

12. Robinson NC, Strey F, Talbert L. Investigation of the essential boundary laeyr phopholipids of cytochrome c oxidase using Triton X-100 delipidation. Biochemistry 1980;19:3656-61.

13. Sedlák E, Robinson NC. Removal of bound cardiolipin destabilizes the quaternary structure of bovine heart cytochrome c oxidase. Biochim Biophys Acta (Suppl.) 2002;12:119

14. Sedlák E, Robinson NC. Phospholipase A2 digestion of cardiolipin bound to bovine cytochrome c oxidase alters both activity and quaternary structure. Biochemistry 1999;38:14966-72.

15. Tsukihara T, Aoyama H, Yamashita E et al. The whole structure of the 13-subunit oxidized cytochrome c oxidase at $2.8 \mathrm{~A}^{\circ}$. Science 1996;272:1136-44.

16. van Gelder BF. Optical properties of cytochromes from beef heart mitochondria, submitochondrial vesicles, and derived preparations. Methods Enzymol 1978; $53: 125-8$

17. van Holde KE, Weischet WO. Boundary analysis of sedimentation - velocity experiments with monodisperse and paucidisperse solutes. Biopolymers 1978; 17:1387-1403

18. Wikstrőm M, Krab K, Saraste M. Cytochrome Oxidase: A synthesis, London: Academic Press, 1981:136.

Doc. RNDr. Jana Staničová, Ph.D., University of Veterinary Medicine, Institute of Chemistry, Biochemistry, and Biophysics, Komenského 73, 04181 Košice, Slovak Republic. e-mail: stanicova@uvm.sk 